\title{
Fibre and Waveguide Lasers
}

\author{
Anne Tropper \\ Physics department, University of Southampton, UK
}

1 Introduction

2 Principles of guided-wave laser devices

2.1 Guided-wave propagation and cut-off

2.2 Beam quality of guided modes

2.2 Gain in active waveguides

2.3 Laser waveguide fabrication techniques

2.4 Fibre and planar laser devices

3 Diode array and bar pumped waveguides

3.1 Brightness conversion and cladding pumping

3.2 Diode-pumped planar waveguide sources

3.3 Mode control in planar waveguide resonators

3.3.1 Mode control in the guided direction

3.3.2 Mode control in the unguided plane

3.4 Face-pumped planar lasers

$4 \quad$ References 


\section{Introduction}

The guided-wave laser is almost as old as the laser itself. The first demonstration of laser action in glass made use of a multimode waveguide; a core rod encased in a cladding of lower refractive index so that confinement of the light would counteract the effect of the poor optical quality of the available glass, (Snitzer, 1961). However, although waveguiding has long been an essential feature of semiconductor diode lasers, in dielectric laser media by far the greatest research effort has gone into the development of bulk rods and slabs of high optical quality. Interest in the potential advantages of guided wave dielectric gain media was only quickened with the advent of high quality single mode optical waveguides, especially rare earth doped silica fibres, in which the propagation losses are so low that the benefits of optical confinement are fully realised.

The most immediate advantage of the guided-wave geometry is that of reducing the cavity mode volume, and hence the pump power needed to reach threshold. Provided the guiding structure is designed so as to support only a single propagating mode at the gain wavelength, then the laser output will be spatially coherent, in a mode that is not affected, for example, by cavity misalignment. The guided-wave laser can be designed as a compact, stable, monolithic device, exploiting all the techniques of integrated optics, such as gratings, couplers, and modulators. Since the active region of a guided-wave laser is typically only a few $\mu \mathrm{m}$ in diameter, fabrication can involve a range of deposition techniques very different from those used to grow bulk media. The resulting gain medium may have a composition or dopant concentration not available to a bulk phase. On the other hand the advantages of a guided-wave structure are cancelled if propagation losses compete too effectively with the achievable gain. A further difficulty attending these optically pumped devices is the need to couple pump light into the waveguide core. The pump sources themselves must therefore emit spatially coherent beams, and expensive micropositioning techniques are required.

The literature on fibre and planar dielectric waveguide lasers is now so extensive that a review of this type cannot attempt to be comprehensive. My aim is rather to sketch the principles of guided-wave laser design and operation, and introduce a few selected devices of particular current interest. I shall pay particular attention to the role that guided-wave systems may play in the effort to develop compact and efficient sources emitting high power diffraction-limited beams. Diode-bar lasers emitting many tens of watts are now readily available, but it remains a challenge to convert the highly asymmetric and multimode output from such a device into a usable beam in a simple and efficient way. It is not at first sight obvious that a guided wave laser should be particularly suitable for high power operation. Waveguides are characteristically devices in which high core intensity accompanies low overall power, and scaling up the core area leads to multimode propagation and loss of spatial coherence. Recently, however, the technique of cladding pumping of fibre lasers has been found to be strikingly effective in overcoming these limitations.

It can be argued that planar waveguide structures are inherently highly compatible with highpower diode-bar pump lasers. Experimental investigation of such devices indicates that extremely compact sources, potentially able to handle $10 \mathrm{~W}$ or more of output, can be fabricated in this way. Control of the spatial mode is a central and difficult problem, and various technical approaches will be reviewed. Equally stringent is the necessity to couple pump radiation into the guide, whether longitudinally for greater efficiency, or transversely for a less divergent output and the possibility of power scaling. We shall see that with careful positioning this can be 
achieved without the use of any optical components whatsoever. If, moreover, the claddingpumping principle is employed, then the position tolerances are significantly relaxed. Alternatively it may be possible to pump through the face of the device, and we shall review some practical schemes of this type.

\section{Principles of guided-wave laser devices}

There exist excellent texts which give an exhaustive account of waveguide modes (e.g. Snyder and Love, 1983; Lee, 1986; Nishihara, Haruna and Suhara, 1985). It is nevertheless worth summarising the simple underlying principles that can often be applied to yield a reasonable estimate of the performance of a device without recourse to elaborate analysis. In addition, the developer of waveguide sources may be concerned with mode properties such as beam quality, which are not discussed in the classic texts.

The simplest conceivable waveguide is the step-index symmetric planar guide, for which closed form expressions can be derived for all physical properties of interest. The discussion of guidedmode properties will therefore be illustrated with reference to this system. The generalisation of these results to asymmetric and channel guides is straightforward. Although more complex index profiles require numerical analysis, for purposes such as the estimation of lasing threshold it is often sufficient to approximate the interfaces by index steps.

\subsection{Guided-wave propagation and cut-off}

The polarisation eigenmodes of a waveguide structure are in general quite unlike those of free space which are purely transverse with respect to both the E- and H-fields. In the step-index planar slab, for example, it is possible for only one of these fields to have no component parallel to the propagation direction of the mode. The non-transverse component describes an ellipse in a plane which contains the propagation direction; the quarter-cycle phase shift between longitudinal and transverse components ensures that only the longitudinal component of the Poynting vector survives time-averaging, and there is no loss of power from the confined mode. Both the aspect ratio and the sense of rotation of this ellipse vary rapidly as a function of position across the mode profile.

From the viewpoint of the waveguide laser designer, who may wish, for example, to minimise the volume of a mode, it is particularly useful to bear in mind the spatial scaling properties of laser modes. The quantity $\lambda / \phi$ has the character of a scaling length, where $\lambda$ is the free space wavelength of the propagating light, and $\phi$ is the numerical aperture (NA) of the waveguide. A waveguide with transverse dimension less than about half a scaling length will in general be monomode; all propagating modes other than the fundamental will be cut off. The NA of a waveguide can be expressed as

$$
\phi=\sqrt{\mathrm{n}_{2}^{2}-\mathrm{n}_{1}^{2}}
$$

in terms of $\mathrm{n}_{2}$, the maximum core index, and $\mathrm{n}_{2}$, the cladding index.

To show how the NA, a quantity which emerges from geometrical optics, comes to play such a central role in the electromagnetic theory of guided-wave propagation, we solve the Helmholtz equation for the structure sketched in Fig 1. 
Figure 1. Planar step-index symmetric waveguide.
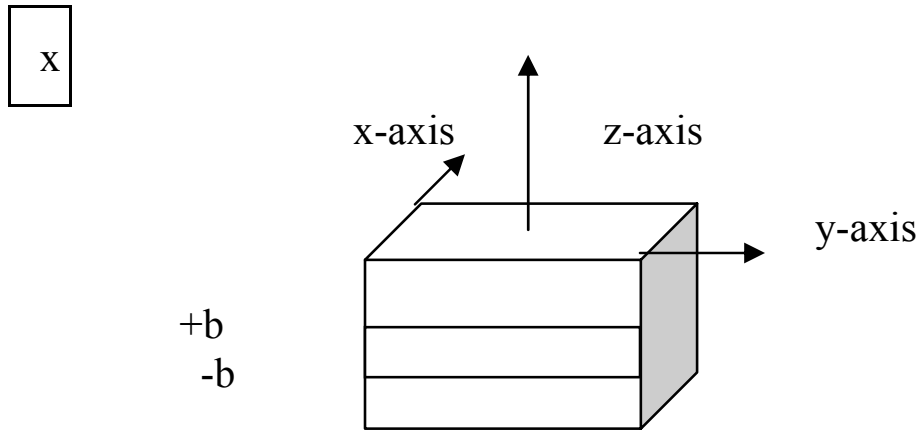

Light propagates along the z-axis guided between core/cladding interfaces in the planes $\mathrm{x}= \pm \mathrm{b}$. The $\mathrm{x}$-dependence of the purely transverse field amplitude of the guided solutions is tabulated in Table 1. It is assumed that the fields are independent of $y$, and have everywhere the plane-wave $\mathrm{z}$-dependence $\exp \left(\mathrm{ik}_{\mathrm{z}} \mathrm{z}\right)$.

Table 1. Modes of a symmetric planar waveguide of depth $2 b$

\begin{tabular}{|l|l|}
\hline core: $\mathrm{n}_{2,}|x|<\mathrm{b}$ & cladding: $\mathrm{n}_{1,}|\mathrm{x}|>\mathrm{b}$ \\
\hline $\begin{array}{l}\text { even } \quad \cos (\mathrm{kx}) \\
\text { odd } \quad \sin (\mathrm{kx})\end{array}$ & $\begin{array}{l}\cos (\mathrm{kb}) \exp (-\mathrm{a}(|\mathrm{x}|-\mathrm{b})) \\
\sin (\mathrm{kb}) \exp (-\mathrm{a}(|\mathrm{x}|-\mathrm{b}))\end{array}$ \\
\hline
\end{tabular}

These are solutions of the Helmholtz equation only if $\mathrm{k}$ and $\mathrm{k}_{\mathrm{z}}$, respectively the transverse and longitudinal propagation constants of the guided mode, and $\mathrm{a}$, the attenuation constant of the evanescent part of the mode are related by

$$
\mathrm{k}^{2}+\mathrm{k}_{\mathrm{z}}^{2}=\mathrm{n}_{2}^{2} \mathrm{k}_{0}^{2} \quad \mathrm{k}_{\mathrm{z}}^{2}-\mathrm{a}^{2}=\mathrm{n}_{1}^{2} \mathrm{k}_{0}^{2}
$$

where $\mathrm{k}_{0}$ is the free space wavevector of the light. It follows that $\mathrm{k}$ and a satisfy

$$
\mathrm{k}^{2}+\mathrm{a}^{2}=\mathrm{k}_{\mathrm{c}}^{2}
$$

where the constant $\mathrm{k}_{\mathrm{c}}$, given by $\phi \mathrm{k}_{0}$, represents an upper limit on the value of the transverse wavevector. A mode for which $\mathrm{k}=\mathrm{k}_{\mathrm{c}}$ has a vanishingly small value of $\mathrm{a}$; its evanescent wings extend indefinitely far into the cladding and it is no longer confined. A key characteristic of any propagating mode is its nearness to this cut-off point, represented by the modal parameter $\delta$. It is convenient to define $\delta$ such that

$$
\mathrm{k}=\mathrm{k}_{\mathrm{c}} \cos (\delta \pi / 2) \quad \mathrm{a}=\mathrm{k}_{\mathrm{c}} \sin (\delta \pi / 2)
$$

The entire range of modal behaviour is therefore spanned as $\delta$ takes values between 0 and 1 . As $\delta$ approaches 0 the mode goes into cut-off. As $\delta$ approaches 1 the mode acquires more of a bulk character; it does not extend outside the core and its polarisation is TEM.

A guidance condition restricting the value of $\delta$ for a given guide thickness, $2 b$, is imposed by the 
electromagnetic boundary conditions, according to which the transverse components of $\mathrm{E}$ and $\mathrm{H}$ must be continuous across the boundaries of the core. For a TE mode this is equivalent to requiring that $\mathrm{E}$ and $(1 / \mu) \mathrm{dE} / \mathrm{dx}$ are continuous. At optical frequencies the magnetic permeability $\mu$ differs negligibly from its free space value, and the guidance condition may be solved to give a closed form expression for the guide thickness:

$$
2 \mathrm{~b}(\delta, \mathrm{m})=\left(\frac{\lambda}{\phi}\right) \frac{\delta+\mathrm{m}}{2 \cos (\delta \pi / 2)} \quad \text { Eq.2.1.1 }
$$

where the mode index $\mathrm{m}$ is a positive integer or zero. The guide thickness is multiple-valued because the guide will in general support both fundamental $(\mathrm{m}=0)$ and higher order modes; these have even or odd parity determined by whether $\mathrm{m}$ is even or odd. The mth mode is just at cut off for a guide of thickness $(\lambda / \phi) \times(\mathrm{m} / 2)$.

The TM modes do not scale rigorously with numerical aperture in this way because the continuous quantities in this polarisation are $\mathrm{H}$ and $(1 / \varepsilon) \mathrm{dH} / \mathrm{dx}$, and in a transparent medium the dielectric response function $\varepsilon$ is the square of the refractive index. The TM guidance condition is found to take the form:

$$
2 \mathrm{~b}(\delta, \mathrm{m})=\left(\frac{\lambda}{\phi}\right) \frac{\Delta+\mathrm{m}}{2 \cos (\delta \pi / 2)} \quad \text { Eq.2.1.2 }
$$

with $\Delta=(2 / \pi) \arctan \left(\mathrm{n}_{2}^{2}(\delta \pi / 2)^{2} / \mathrm{n}_{1}^{2}\right)$.

Figure 2: Depth of a symmetric waveguide, units of $(\lambda / \mathrm{NA})$, as a function of guidance parameter $\delta$ for TE and TM modes with mode integer $\mathrm{m}=0,1,2,3,4,5$.

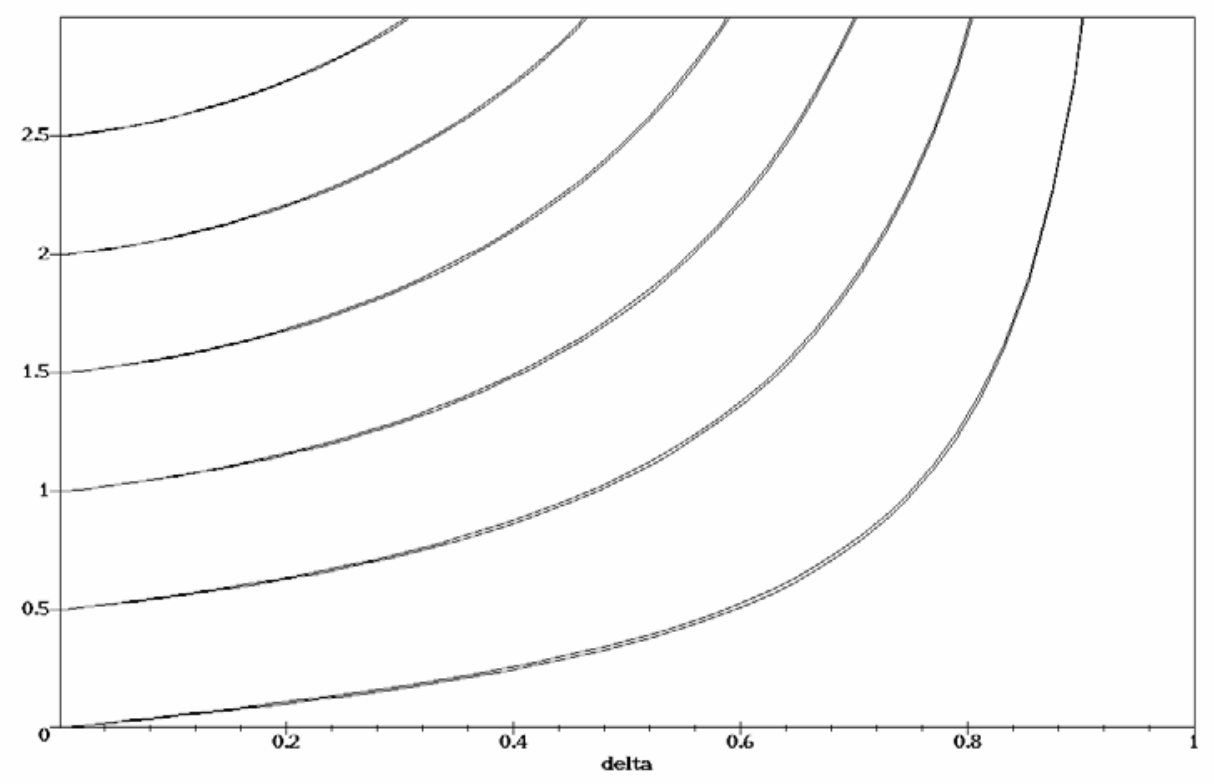


Fig 2 shows the dependence of the guide thickness, in units of the scaling length, with the modal parameter $\delta$ for several TE and TM modes, calculated from Eqs. 2.1.1 and 2.1.2. This is much quicker than solving for $\delta$ numerically, and the curve can be used to look up values of $m$ and $\delta$ for any guide of known thickness. The TE curves are universal in that they apply to any combination of core and cladding indices. The TM curves are specific to the values $\mathrm{n}_{1}=1.5$ and $\phi=0.4$, however it can be seen that the TM modal parameters are quite close to their TE values even for this extreme index difference. The TM mode is always very slightly closer to cut-off than the TE mode. For some practical purposes the difference is not significant, however it can be exploited, for example in the use of metal overlayers to make waveguide polarizers. In section 3.2 of this chapter an application of this effect to spatial mode control in planar waveguide lasers will be discussed.

The eigenmodes of a circular fibre waveguide with a step-index profile can also be written in a closed form (Snyder and Love 1982), albeit a rather complex and unattractive one. The polarization properties are particularly complicated, and both $\mathrm{E}$ and $\mathrm{H}$ will have longitudinal components unless the mode exhibits azimuthal symmetry. For very many practical devices, however, it is valid to analyse the modes in the weak-guidance approximation:

$$
\Delta \mathrm{n}=\mathrm{n}_{2}-\mathrm{n}_{1}<<\mathrm{n}_{2}
$$

The radial part of the intensity distribution of each mode then resembles the form given for the step index guide in Table 1, with the sine/cosine and exponential functions replaced respectively by linear combinations of oscillatory Bessel functions of the first and second kind, and by modified Bessel functions of the first kind. In this approximation the modes can be designated $\mathrm{LP}_{\mathrm{qm}}$ and their polarisation is close to TEM. The first mode integer q refers to the dependence of the intensity on the azimuthal angle $\chi$, which may be either $\cos ^{2}(q \chi)$ or $\sin ^{2}(q \chi)$. The $\mathrm{LP}_{0 \mathrm{~m}}$ modes are plane polarised. An active fibre device is ideally designed to support only the fundamental $\mathrm{LP}_{01}$ mode at the operating wavelength. For a step-index fibre this requires $\mathrm{V}<$ 2.401 where the dimensionless parameter $\mathrm{V}$ is simply the fibre radius, $b$, measured in units of $(\lambda / \phi)$ and multiplied by $(2 \pi)$. Provided the fibre NA is low, and $V>1$, the intensity profile of the $\mathrm{LP}_{01}$ mode approximates closely to a Gaussian function:

$$
\mathrm{I} \approx \exp \left[-\frac{\mathrm{r}^{2}}{\mathrm{r}_{0}^{2}}\right] \quad \text { where } \mathrm{r}_{0}=\frac{\mathrm{b}}{\sqrt{(2 \ln \mathrm{V})}}
$$

whence one can estimate that a fraction $\left(1-1 / \mathrm{V}^{2}\right)$ of the modal power will be confined within the fibre core.

\subsection{Beam quality of guided modes}

The form of the mode profile changes strikingly as the mode approaches cut-off. This is illustrated in Figure 3, which shows the $x$-variation of the E-field of a TE $m=4$ mode for different values of the guide thickness. 
Figure 3: E-field amplitude of a symmetric waveguide TE mode with $\mathrm{m}=4$ as a function of $\mathrm{x}$ measured in units of $(\lambda / \phi)$. The curves represent a series of waveguides of decreasing depth, supporting modes for which $\delta=0.9,0.8,0.7,0.6,0.5,0.2$ and 0.02 .

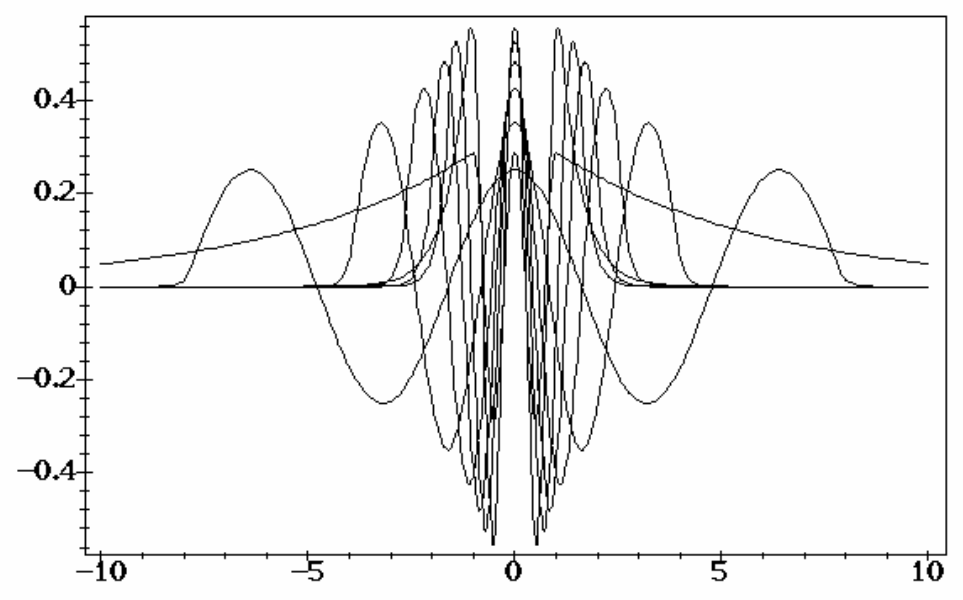

The amplitudes are normalised so that each curve corresponds to the same total power. As the guide gets thinner the peak amplitude initially grows with increasing confinement of the light and then drops away as the evanescent wings extend out and the mode goes into cut-off. In this regime the mode profile is strikingly unlike that of any free space propagation eigenmode, raising a general question about the beam quality of guided wave lasers.

The 'quality' of a paraxial beam is usually characterized by the $\mathrm{M}^{2}$ parameter, which quantifies by how many times the beam divergence exceeds the fundamental limit set by diffraction. For non-Gaussian beams we need a more general measure of beam radius than the $1 / \mathrm{e}^{2}$ intensity point. Such a measure is supplied by the square root of the variance in $\mathrm{x}$, defined by

$$
<\mathrm{x}^{2}>=(1 / \mathrm{N}) \int_{-\infty}^{+\infty} \mathrm{x}^{2}|\mathrm{E}(\mathrm{x})|^{2} \mathrm{dx} \quad \text { where } \mathrm{N}=\int_{-\infty}^{+\infty}|\mathrm{E}(\mathrm{x})|^{2} \mathrm{dx}
$$

The corresponding measure of the beam divergence is the variance in the transverse wavevector, $<\mathrm{k}^{2}>$. Let $F(\mathrm{k})$ be the amplitude spectrum of spatial frequencies in the beam, i.e. the 1dimensional Fourier Transform of E(x). Then we can define

$$
<\mathrm{k}^{2}>=(1 / \mathrm{N}) \int_{-\infty}^{+\infty} \mathrm{k}^{2}|F(\mathrm{k})|^{2} \mathrm{dk}=(1 / \mathrm{N}) \int_{-\infty}^{+\infty}\left|\frac{\partial \mathrm{E}}{\partial \mathrm{x}}\right|^{2} \mathrm{dx}
$$

The variance in $\mathrm{k}$ can be related to an effective NA for the beam:

$$
(\mathrm{NA})^{2}=\frac{<\mathrm{k}^{2}>}{\mathrm{k}_{0}^{2}}
$$

The quality of a beam is conveniently characterized by the $\mathrm{M}^{2}$-parameter, which quantifies how many times the beam divergence exceeds the fundamental limit set by diffraction. For any paraxial beam

$$
<\mathrm{k}^{2}><\mathrm{x}_{0}^{2}>=\frac{\left(\mathrm{M}^{2}\right)^{2}}{4}
$$

where $<\mathrm{x}_{0}^{2}>$ corresponds to the narrowest point of the beam at the waist. The left-hand product 
takes its minimum value when $\mathrm{M}^{2}=1$ and the beam is diffraction-limited.

It is therefore straightforward to calculate the $\mathrm{M}^{2}$-value of a beam emitted from the end of a waveguide when the distribution of E-field over the end face corresponds to a known eigenmode of the structure. In Fig. 4 the variation of $\mathrm{M}_{\mathrm{x}}{ }^{2}$ with the mode parameter $\delta$ is plotted for the first few even modes of a symmetric step-index planar guide. It can be seen that the fundamental mode is nearly diffraction-limited, with $\mathrm{M}^{2}<1.1$, over a wide range of values of $\delta$. A striking feature of the behaviour is that the $\mathrm{M}^{2}$-value of each higher-order mode diverges as the mode approaches cut-off. The beam quality of a guided-wave laser, which emits mostly fundamental mode light, may therefore be significantly degraded by the admixture of a small amount of a higher order mode close to cut-off.

Figure 4: $\mathrm{M}_{\mathrm{x}}{ }^{2}$ as a function of $\delta$ for even modes of a symmetric waveguide with $\mathrm{m}=0,2,4,6$.

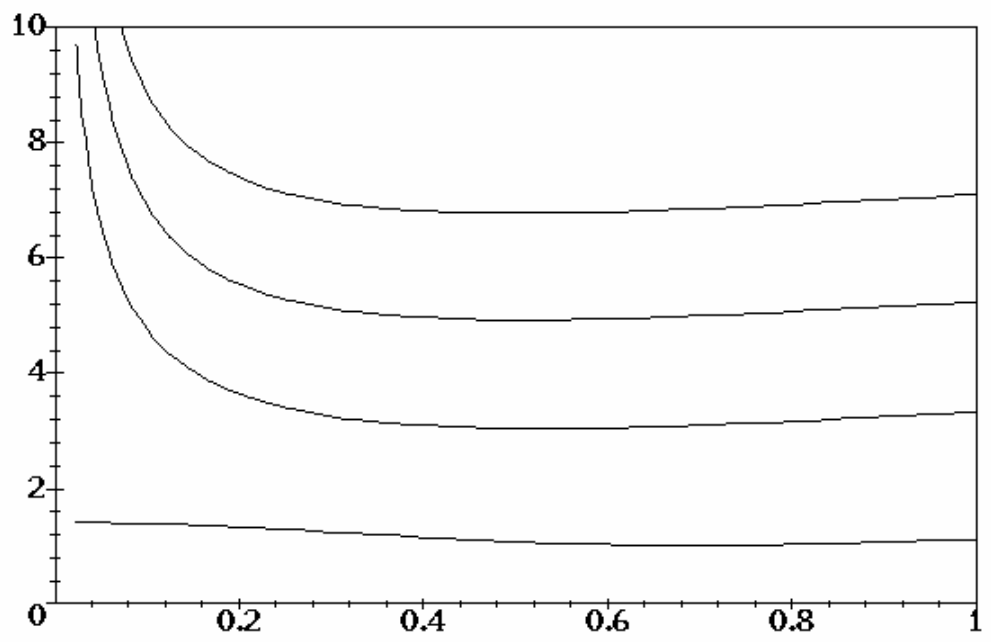

\subsection{Gain in active waveguides}

Many dielectric laser systems are pumped longitudinally, often by diode lasers. It is interesting to consider whether more efficient conversion of the available pump power can be achieved by fabricating a waveguide in the gain medium. The aim is to reduce the threshold of the device roughly by the factor by which optical confinement reduces the volume of the lasing mode in the gain medium.

The length, 1 , of the gain medium in a longitudinally-pumped laser is determined by the strength of the pump absorption. Assuming a Gaussian cavity mode confocally focussed in the gain medium, the mode volume in the medium will be $\sim \lambda 1^{2}$. If the cavity mode is confined with respect to one axis in a planar guide of numerical aperture $\phi$, the mode volume is reduced relative to its bulk value by a factor of $\alpha=\phi(1 / \lambda)^{1 / 2}$. If the mode is confined with respect to both axes, as in a fibre or channel guide, its volume is reduced by a factor of $\alpha^{2}$. The usefulness of guidedwave designs is therefore greatest in weakly absorbing media, as strikingly exemplified by rare earth doped silica fibre lasers. Coefficients of absorption and gain per unit length in such fibres may be only $\sim 0.01 \mathrm{~cm}^{-1}$, with fibre lengths of many metres and $\alpha$-values of several hundred. It 
is clear why these devices have no bulk counterpart.

There is an extensive literature describing the calculation of gain in guided-wave amplifiers; the reader is referred in particular to the work by Desurvire, 1994. A key result (e.g. Digonnet and Gaeta,1985) relates the unsaturated single-pass gain exponent, $\gamma$, to an effective pump area, $A_{\text {eff: }}$ :

$$
\gamma=\frac{\sigma \tau}{\mathrm{h} v_{\mathrm{p}}} \frac{\mathrm{P}_{\mathrm{abs}}}{\mathrm{A}_{\mathrm{eff}}} \quad \text { Eq.2.3.1 }
$$

where $\mathrm{P}_{\mathrm{abs}}$ is the total power absorbed from the pump beam in the gain medium, $\mathrm{h} \mathrm{v}_{\mathrm{p}}$ is the pump photon energy, and $\sigma$ and $\tau$ are the emission cross-section and upper state lifetime respectively. The effective pump area is given by:

$$
\mathrm{A}_{\text {eff }}^{-1}=1 \int_{\text {volume }} \mathrm{R}(\mathrm{r}) \mathrm{S}(\mathrm{r}) \mathrm{d}^{3} \mathrm{r} \quad \text { Eq.2.3.2 }
$$

where 1 is the length of the amplifier, and $\mathrm{R}, \mathrm{S}$ are normalized pump-rate and signal-mode intensity distributions. In a monomode fibre or channel guide $\mathrm{A}_{\mathrm{eff}}$ is of order the square of the scaling length. $(\lambda / \phi)^{2}$; its precise value is determined by the degree of overlap of pump and signal modes.

In a device with a small effective area it is often possible to invert a transition strongly at quite moderate values of pump power. Eqs. 2.3.1 and 2.3.2 may then give a misleading overestimate of the gain, partly because saturation of the pump absorption reduces the effective overlap of pump and signal and also because parasitic effects such as upconversion may degrade the quantum efficiency of the pumping process (Guy et al 1998).

\subsection{Laser waveguide fabrication techniques}

The single most important class of active dielectric waveguide is undoubtedly that of silica fibres fabricated by chemical vapour deposition. These structures may be based on the $\mathrm{Ge}_{2} \mathrm{O}_{3}-\mathrm{SiO}_{2}$, $\mathrm{Al}_{2} \mathrm{O}_{3}-\mathrm{SiO}_{2}$, or $\mathrm{P}_{2} \mathrm{O}_{5}-\mathrm{SiO}_{2}$ glass systems, with NA in the range $0.1-0.4$, and propagation loss as low as $\sim 1 \mathrm{~dB} \mathrm{~km}^{-1}$. The erbium-doped fibre amplifier exemplifies all the most desirable properties of gain media of this type; smooth broad spectral features, low dopant concentration with negligible ion-ion interaction, low background loss, great mechanical robustness, and efficient amplification.

The excellent properties of silica glass fibre as a laser host medium have turned out to be actually rather specific to just a few rare earth ion laser transitions in the near-infrared region of the spectrum. The high frequency vibration modes of the silica matrix render the fibres absorbing at longer wavelengths, and also tend to quench the inversion on shorter wavelength laser transitions. Thus most silica fibre laser development involves one of three systems: 1) the 2- $\mu \mathrm{m}$ $\mathrm{Tm}^{3+}$ transition is of interest for sensor applications, 2) the $0.98-1.1 \mu \mathrm{m} \mathrm{Y \textrm {Y } ^ { 3 + }}$ transition, well suited to high power applications both because the difference between the pump and laser photon energies is small, reducing thermal load, and because the simple level structure of $\mathrm{Yb}^{3+}$ confers freedom from parasitic cross-relaxation processes, and 3) the $1.55-\mu \mathrm{m} \mathrm{Er}^{3+}$ transition, whose importance in telecommunications and as an eye-safe transition is well known. Er-doped silica fibre often includes large concentrations of $\mathrm{Yb}$ as a co-dopant ion which will absorb 1064-nm pump radiation and transfer it efficiently to the Er population, so that devices can be made short.

These three transitions have similar character; each spans the unusually large energy gap which 
strong spin-orbit coupling produces between the ground and first excited level manifolds in these rare earth ions with nearly filled $4 \mathrm{f}$ shells. Since there is no exact point symmetry at the site of a dopant ion in a glass matrix, there are also no strong selection rules, and the transition dipole moment is more or less evenly distributed between the individual transitions linking the manifolds. In the Er transition, for example, there are 56 of these, since the upper and lower manifolds contain 7 and 8 Kramers doublet levels respectively. It tends to be characteristic of the energy eigenvalues of high angular momentum ions in approximately octahedral fields that one level of a manifold lies well below the others, which are clustered relatively closely in energy (Yeung and Newman, 1985). This phenomenon confers the characteristic fluorescence profile, seen especially in the emission of $\mathrm{Er}$ and $\mathrm{Yb}$, in which the lowest-to-lowest transition forms a partially resolved spike at the high energy extremity of the band, and all the other transitions combine to form a broad featureless wing extending to lower energies. The transition wing is in effect homogeneously broadened, the dense clustering of many transitions tending to obscure the inhomogeneous site-to-site variation of individual transition frequencies.

Within each manifold of levels the population relaxes to a Boltzmann distribution at the glass matrix temperature within a few ps; this process is fast compared to the rate at which population relaxes between different manifolds, whether radiatively or by multiphonon emission. There is as a result a proportional relationship between the emission and absorption cross-sections at any frequency within the transition bandwidth which has often been used, for example, to extract emission cross-section values from absorption measurements (Miniscalco and, Quimby, 1991). An important consequence of the fast thermalisation of population within the upper and lower manifolds of levels is that the corresponding laser transition varies smoothly from 3-level to 4level as the transition wavelength increases. However it is often the case that the pump saturation power required to halve the total population of the ground state manifold is only a few tens of $\mathrm{mW}$ or less in a monomode fibre, so that 3-level character is quite compatible with high efficiency.

"Soft glass" fibres, in which the vibration spectrum cuts off at energies $<<1200 \mathrm{~cm}^{-1}$, are potentially more versatile than silica; however the materials problems which these structures present has prevented exploitation of their properties. The best-established soft fibre technology at the present time is based on a fluorozirconate glass-forming system; $\mathrm{ZrF}_{4}-\mathrm{BaF}_{2}-\mathrm{LaF}_{3}-\mathrm{NaF}$, or "ZBLAN". There is no CVD technology for fluoride glass, so preforms of these fibres are fabricated by a rotational casting technique. Fibre losses are typically at the $\mathrm{dB} \mathrm{m}^{-1}$ level, and numerical apertures up to 0.4 have been achieved. ZBLAN fibres are far more fragile than their silica counterparts, and they are potentially vulnerable to chemical attack by water. There is substantial published work on the applications of these fibres as laser sources in the $2-4 \mu \mathrm{m}$ region, and also at visible wavelengths.

One other glass fibre system has recently become commercially available after some years of development by several groups. Chalcogenide fibres with extended transparency in the infrared offer some interesting device prospects (Wang et al, 1997), but are even more chemically unstable and mechanically fragile than ZBLAN.

With regard to planar waveguide fabrication the situation is more complicated because no single indispensable technology has emerged comparable to that of the EDFA, although at least 9 distinct techniques have been used to realise planar and channel lasers. Research in this field has been motivated by the desire to make more compact devices than glass fibre allows, to 
miniaturise vibronic lasers for which glass hosts do not exist, and to make fully integrated modelocked and Q-switched sources in a monolithic format.

Much published work relates to chemical surface treatments of glass or crystalline substrates, involving either ion exchange or thermal indiffusion. A planar glass technology of interest for wavelength-division-multiplexing devices in telecommunications is ion-exchange; guides of NA $>0.5$ can be made by replacing $\mathrm{Na}^{+}$ions in the substrate with $\mathrm{Tl}$ ions from a bath of molten salt. Proton exchange is a classic technique of integrated optics on lithium niobate substrates, and it has also been used successfully to make laser devices. Only the extraordinary index of the uniaxial crystal is modified by the exchange, limiting the usefulness of the technique to pipolarised laser transitions; but guides of NA as high as 0.7 result.

Some work on epitaxial growth techniques can also be found in the literature. Crystalline guides of notably high quality based on $\mathrm{Y}_{3} \mathrm{Al}_{5} \mathrm{O}_{12}$ have been grown by liquid phase epitaxy (LPE). In this work the active layer was doped with Ga to raise the index and Lu to achieve a lattice match.

$\mathrm{An} \mathrm{Yb}$-doped structure of this kind operated as a quasi-3-level laser with near quantum-limited efficiency owing to good optical confinement and low waveguide propagation losses of $\sim 0.1 \mathrm{~dB}$ $\mathrm{cm}^{-1}$ (Pelenc et al, 1995). Structures with NA up to 0.2 have been made in this way, however the Ga doping has the effect of broadening the linewidth of the laser transitions, thereby reducing the gain by a factor of $\sim 2$. More recently the fabrication by molecular beam epitaxy (MBE) of crystalline fluoride waveguides has been developed (Daran et al., 1998), and led to the demonstration of lasing in a planar Nd-doped $\mathrm{LaF}_{3}$ guide. This system may allow a planar technology of diode-pumped sources for the mid-infrared to be developed.

Many groups have worked to develop physical processes of waveguide deposition that might be applicable to a wide range of material systems. An early example was high-energy helium ion implantation, which was indeed used to create waveguides in numerous crystals and glasses (Townsend, 1990). Many of these structures exhibited guided-wave lasing; alas the propagation losses of the resulting guides in most cases badly compromised their performance as lasers. Subsequent work, however, suggests that these losses are not intrinsic to the implantation process, and may be substantially reduced by taking greater care over the prior preparation of the implanted surface (Peto et al., 1997). More recently pulsed laser deposition (PLD) has been reported as a technique by which low loss crystalline garnet guides can be made (Bonner et al., 1997).

Vibronic laser transitions have long interested the waveguide laser community, since their inherently high threshold can in principle be markedly reduced in an optically confined structure. To be successful such a device must incorporate a high quality waveguide with low propagation loss, since the gain coefficients of these enormously broad transitions are correspondingly small. Two examples of guided-wave titanium-doped sapphire lasers have recently been reported; one fabricated by PLD on a sapphire substrate (Anderson et al., 1997) and the other fabricated by thermal indiffusion of titanium ions into the surface of a sapphire crystal (Hickey et al., 1998).

A particularly versatile and promising fabrication technique is thermal bonding, involving simply the contacting of surfaces which have been polished to an optical finish and chemically cleaned. In the low temperature version of this technique the surfaces are held together by van der Waals forces, and are not chemically modified. Chemically and structurally dissimilar materials can be bonded, with only the proviso that the thermal expansion coefficients of substrate and superstrate 
must be compatible. Once the bond is formed the composite structure is strong enough to allow the superstrate to be mechanically thinned by polishing, even down to the size required for the core of a monomode waveguide. An upper cladding layer can be attached in a second bonding step. Even channel guides can in principle be fabricated. Planar laser guides made in this way have been shown to exhibit propagation losses of $\sim 0.16 \mathrm{~dB} \mathrm{~cm}^{-1}$, deduced from measurements of laser slope efficiency as a function of output coupling (Brown et al.1997). Composite guides have been reported with numerical aperture values spanning a range from 0.06 (Nd-doped YAG core and undoped YAG cladding) to 0.8 (Nd:YAG core and glass cladding).

\subsection{Examples of fibre and planar devices}

A key technology in the development of silica fibre laser sources is the fibre Bragg grating, used to select specific operation wavelengths out of broad gain spectra, to enforce lasing in the far wings of a transition, as a notch filter, as a dispersion compensator and in conjunction with fibre couplers to configure a variety of complex and versatile laser cavities. The grating consists of a fibre region over which the index of the core is periodically or aperiodically modulated. A grating may be fabricated by exposing a photosensitive fibre to ultraviolet light through an appropriate phase mask (Hill et al., 1993). An early application of the fibre grating was to the development of a distributed feedback Er-Yb fibre laser, emitting a single longitudinal mode with a frequency bandwidth of $300 \mathrm{kHz}$ at $1534 \mathrm{~nm}$ (Kringlebotn et al., 1994).

Whereas the UV-written fibre grating has static phase properties, acoustic gratings can be used to make dynamic Bragg-coupled elements. A fibre acousto-optic frequency shifter consists of a four-port fused taper coupler made with dissimilar fibres that are so phase mismatched that coupling is zero in the absence of an acoustic wave driven into the coupling region (Birks et al, 1994). The coupled light is frequency-shifted, and some tuning of the acoustic frequency is possible. An example of the intriguing possibilities of this technique is provided by the slidingfrequency Er-Yb soliton laser. A ring cavity contains an Er-Yb-doped gain section and an acoustically-driven 4-port coupler of the type described above, which acts both as frequency shifter and as tunable bandpass filter. The laser exhibits self-starting modelocking; it prefers to pulse because cw radiation at the gain peak is progressively detuned and sees greater loss than solitons which are spectrally reshaped as they circulate (Culverhouse et al, 1995).

The all-fibre cavity of this laser bears little resemblance to a traditional laser resonator; its several components are connected by fusion splices and need no further optical alignment. Conceptually similar types of resonator are constructed using planar technology, only in place of metres of fibre wrapped on drums a few $\mathrm{cm}^{2}$ of substrate will accommodate the cavity components. A harmonically mode-locked Ti:Er: $\mathrm{LiNbO}_{3}$ waveguide laser has been reported which emits transform-limited pulses $3.8 \mathrm{ps}$ in length from a device $54 \mathrm{~mm}$ long (Suche et al, 1995).

The development of high-quality fluorozirconate fibres allowed the first upconversion fibre lasers to be realised in the early nineteen nineties (Allain et al 1991, Smart et al, 1991); visible lasers which could be pumped in the infrared at convenient diode laser wavelengths by sequential ground and excited state absorption steps. Many upconversion fibre lasers based on ZBLAN have been reported, including transitions at violet and ultraviolet wavelengths (Funk and Eden, 1995), however many of these require pumping at a difficult wavelength, or even dual 
wavelength pumping. One attractively simple and versatile scheme uses the $\mathrm{Pr}^{3+}$ ion, which has visible laser transitions at $636 \mathrm{~nm}, 615 \mathrm{~nm}, 520 \mathrm{~nm}$ and $491 \mathrm{~nm}$, all originating from the same metastable level. This level can be pumped by $860-\mathrm{nm}$ light in a fibre co-doped with Pr and Yb; the $\mathrm{Yb}$ ions transferring energy efficiently into the Pr system (Xie and Gosnell, 1995). A particularly efficient 480-nm blue upconversion laser in Tm-doped ZBLAN fibre was first reported by Grubb (1992). A fortuitous overlap of two excited state and one ground state absorption bands allowed this laser to be pumped at a single wavelength in the $1.12-1.14 \mu \mathrm{m}$ range; Grubb reported a 32\% slope efficiency with respect to absorbed pump power. Direct diode pumping has been used to achieve $102 \mathrm{~mW}$ output power on this transition (Sanders et al, 1995 ), and with a high power bar-pumped Nd:YAG laser as the pump source $230 \mathrm{~mW}$ of output were observed (Paschotta et al, 1997).

The most attractive feature of diode-pumped upconversion fibre lasers as all-solid-state cw visible laser sources is their simplicity. No phase-matching is involved, and the broad excitation bandwidth confers a relaxed specification on the spectral characteristics of the pump diode. Although these devices are in principle spatially multimode, since the same core structure guides both signal light and pump light of much longer wavelength, in practice the emitted beam is close to diffraction-limited, presumably because the fundamental mode sees most gain. The exploitation of this technology has, however, been inhibited to date not only by the high cost of ZBLAN fibre, but also by the susceptibility of the fibre to photochromic damage induced by high pump intensities in the core. A particularly acute problem is documented for the Tm system, in which absorption at the blue transition wavelength develops in the presence of infrared pumping at a rate consistent with the formation of colour centres (Barber et al 1995, Laperle et al 1995, Booth et al, 1996, and Paschotta et al, 1997). The detailed behaviour of the fibre appears to be a function of its composition, suggesting that a composition might be found which is resistant to this effect.

\section{Waveguides pumped by high power diodes}

The appearance of commercial diode pump lasers, emitting many tens of watts continuously with great efficiency, has stimulated intense effort into scaling up the power output of diode-pumped solid state lasers, as needed for numerous applications in materials processing, medicine and information technology. The problems inherent in this process are well known. The spatial coherence of a diode bar laser in the plane of the junctions is poor, and it is difficult to use the highly divergent and asymmetric beam efficiently. Moreover a substantial fraction of the pump power absorbed in the gain medium is dissipated thermally. The resulting distributions of temperature and stress will ultimately cause stress fracture of the rod; at lower pump power inputs they give rise to thermal lensing, and birefringence of the laser medium which can severely degrade the spatial quality of the output beam.

A key role for diode-pumped solid state lasers is therefore to convert the power supplied by a bar into a diffraction-limited beam which can be focused, launched into a monomode fibre or used to pump processes of nonlinear frequency conversion to wavelengths of interest. This function is sometimes characterised as brightness conversion, where the term "brightness" has its photometric sense, and may be defined for a paraxial beam in terms of the $\mathrm{M}^{2}$ beam quality parameter introduced earlier as $\mathrm{P} /\left(\lambda^{2} \mathrm{M}_{\mathrm{x}}{ }^{2} \mathrm{M}_{\mathrm{y}}{ }^{2}\right)$, where $\mathrm{P}$ is the power in the beam. Thus whereas the brightness of a gas laser source can easily be many tens of $\mathrm{W} \mathrm{sr}^{-1} \mu \mathrm{m}^{-2}$, the brightness of 
diode bar is typically in the range $10-20 \mathrm{~mW} \mathrm{sr}^{-1} \mu \mathrm{m}^{-2}$.

\subsection{Brightness conversion and cladding pumping}

Consider a conventional fibre laser with a single cladding region. If it is to be pumped with a multimode beam of quality $\mathrm{M}_{\mathrm{p}}{ }^{2}$, which must be focussed to a spot equal roughly in size to the fibre radius $b$, then the fibre must be designed with a numerical aperture at least equal to the pump divergence;

$$
\frac{\mathrm{M}_{\mathrm{p}}^{2} \lambda_{\mathrm{p}}}{\pi \mathrm{b}} \approx \phi
$$

It follows that the fibre $\mathrm{V}$-values at pump and laser wavelengths are given by:

$$
\mathrm{V}_{\text {pump }} \approx 2 \mathrm{M}_{\mathrm{p}}^{2} \quad \mathrm{~V}_{\text {laser }} \approx 2 \frac{\lambda_{\mathrm{p}}}{\lambda_{1}} \mathrm{M}_{\mathrm{p}}^{2}
$$

The laser emission may be usefully improved in spatial coherence relative to the pump beam if its wavelength is significantly longer, and the fundamental mode of the fibre may see more gain and extract the stored energy more effectively. However in general such a device is not a particularly effective brightness converter.

This limitation is dramatically overcome if the fibre is fabricated for cladding pumping, with multimode pump radiation guided in a large area inner cladding region of high $\mathrm{V}$-number. Nested within the inner cladding is a monomode core structure doped with the laser ion. The effective absorption length for the pump radiation is thus increased by approximately the ratio of the cross-section areas of the inner cladding and core regions. An output of $35 \mathrm{~W}$ in a diffractionlimited beam has been reported for a cladding-pumped $\mathrm{Yb}$-doped fibre laser operating at the long wavelength end of the gain bandwidth, where ground state reabsorption is negligible. This device is pumped by low-brightness diodes with a slope efficiency of 65\% (Muendel et al, 1997). A recent review outlines the exciting prospects for compact sources offering high power and high pulse energy using cladding-pumped fibre technology (Richardson et al, 1997).

A cladding-pumped fibre laser achieves brightness conversion by greatly extending the length of the gain medium. This leads to a satisfactorily low thermal loading per unit length, but may impair the efficiency of the laser unless the fibre can be fabricated with sufficiently low propagation losses in the core and inner cladding. In particular there is a trade-off between pump launch efficiency and pumping rate, so that the cladding pumping technique is only likely to perform well for 4-level laser transitions. For those laser transitions in which intense pumping is essential, and stress fracture sets a limit to the thermal loading which can be applied, the slab geometry has often been preferred to the rod. The reason for this is apparent if one compares the temperature differences, $\Delta \mathrm{T}_{\text {rod }}$ and $\Delta \mathrm{T}_{\text {slab }}$ which develop across the rod and the slab respectively, when each experiences a power P/unit length deposited uniformly through its volume:

$$
\Delta \mathrm{T}_{\text {rod }} \approx \frac{1}{4 \pi \kappa} \times \mathrm{P} \quad \Delta \mathrm{T}_{\text {slab }} \approx \frac{1}{2 \kappa} \times \mathrm{P} \times \frac{\mathrm{t}}{\mathrm{W}}
$$

where $\kappa$ is the thermal conductivity of the gain medium and $t$ and $\mathrm{W}$ are the thickness and width respectively of the slab, assumed cooled from one side only. It is thus possible to control the 
temperature excursion using the aspect ratio of the slab. The limiting case of a wide, thin slab is clearly a planar waveguide.

\subsection{Diode-pumped planar waveguide sources}

These ideas motivate an investigation of the potential of planar waveguide lasers as brightness converters for high-power diode bar lasers. Coupling of the diode bar radiation into a planar waveguide structure is a relatively straightforward task: there is no need to symmetrise the diode output, and cylindrical coupling lenses of short focal length and high numerical aperture are readily available. On the other hand, the gain region is correspondingly asymmetric, and it becomes difficult to extract the power in a good quality beam. One of the first experiments of this type therefore used a high-brightness broad-stripe diode to pump a Nd:YAG planar waveguide amplifier. The diffraction-limited signal ensured an output of good beam quality, even though the gain region was $\sim 220 \mu \mathrm{m}$ wide in the unguided plane, but had only the $3.8 \mu \mathrm{m}$ depth of the near single-mode waveguide. Up to $550 \mathrm{~mW}$ of the output from the 1.2-Wdiode was absorbed in the 3.8- $\mu \mathrm{m}$ deep waveguide, fabricated by LPE with a numerical aperture of 0.23 . The dependence of output power on signal input power is shown in Fig. 4 (Shepherd et al 1997). With an input of $90 \mathrm{~mW}$ it was possible to extract $290 \mathrm{~mW}$, or $36 \%$ of the absorbed pump power.

At small signal input power levels the highest measured value of amplifier gain was $28.8 \mathrm{~dB}$. It was shown that this value was significantly reduced by Auger upconversion and cross-relaxation; processes which transfer energy between ions and quench the upper level population (Guy et al, 1998). Thus although it is possible to amplify a diffraction-limited beam efficiently in a planar waveguide, parasitic effects tend to dissipate the high inversion densities that are theoretically attainable in a waveguide. In practice it may be preferable to configure the planar guide as a laser, with the inversion clamped at a moderate value, and use other techniques to control the spatial mode of the output.

A further problem which arises in the attempt to scale planar amplifiers up in power to the multiwatt level is associated with amplified spontaneous emission (ASE). As a rough guide, ASE will saturate the gain of an amplifier above the limit

$$
\frac{\mathrm{G} \Omega}{4 \sqrt{\ln \mathrm{G}}} \approx 1
$$

where $\Omega$ is the solid angle over which the full amplifier gain appears. Consider an amplifier of length 1 , pumped by a multimode beam which has beam quality parameter value of $\mathrm{M}_{\mathrm{y}}{ }^{2}$ in the unguided plane. If the pump is confocally focused with spot size $\mathrm{W}_{\mathrm{p}}$ at the waist in a guide of index $\mathrm{n}$ and numerical aperture $\phi$, the values of $\Omega$ and 1 are given by

$$
\Omega \approx \pi \times \frac{\phi}{\mathrm{n}} \times \frac{\mathrm{M}_{\mathrm{y}}^{2} \lambda_{\mathrm{p}}}{\mathrm{n} \pi \mathrm{W}_{\mathrm{p}}} \quad 1 \approx \frac{2 \pi \mathrm{nW}_{\mathrm{p}}^{2}}{\mathrm{M}_{\mathrm{y}}^{2} \lambda_{\mathrm{p}}}
$$

It follows that the greatest value of gain achievable by such an amplifier, $G_{\max }$, is set by the lateral beam quality parameter of the diode pump source:

$$
\frac{\mathrm{G}_{\max }}{\left(\ln \mathrm{G}_{\max }\right)^{1 / 2}} \approx \frac{2 \mathrm{n}}{\phi \pi} \sqrt{\frac{2 \pi \mathrm{nl}}{\lambda_{\mathrm{p}} \mathrm{M}_{\mathrm{y}}^{2}}}
$$


The value of $\mathrm{M}_{\mathrm{y}}{ }^{2}$ for the broad stripe diode used to pump the amplifier described above is $\sim 34$. The corresponding value of $\mathrm{G}_{\max }$ is $28 \mathrm{~dB}$, showing that this device operates very close to the limit imposed by ASE. One may estimate the value of $\mathrm{G}_{\max }$ achieved by a similar planar amplifier, again with $1 \sim 5 \mathrm{~mm}$ and numerical aperture 0.23 , only now pumped by a more powerful diode bar with a lateral beam quality parameter $\mathrm{M}_{\mathrm{y}}{ }^{2} \sim 2300$. In this case $\mathrm{G}_{\max }$ is only $15 \mathrm{~dB}$, showing that with pump sources of such low brightness it is not feasible to make high gain planar amplifiers by confining the pump light tightly in the direction in which it is spatially coherent.

A first demonstration of a high power planar waveguide source pumped by a diode bar therefore involved a laser rather than an amplifier (Bonner et al., 1998). A simple cylindrical lens assembly was used to couple radiation from a diode bar source into an LPE-grown planar $\mathrm{Nd}$ :YAG waveguide. A diagram of the device is shown in Fig 5.

Figure 5: Diode bar coupled to planar Nd:YAG waveguide by cylindrical lens assembly.

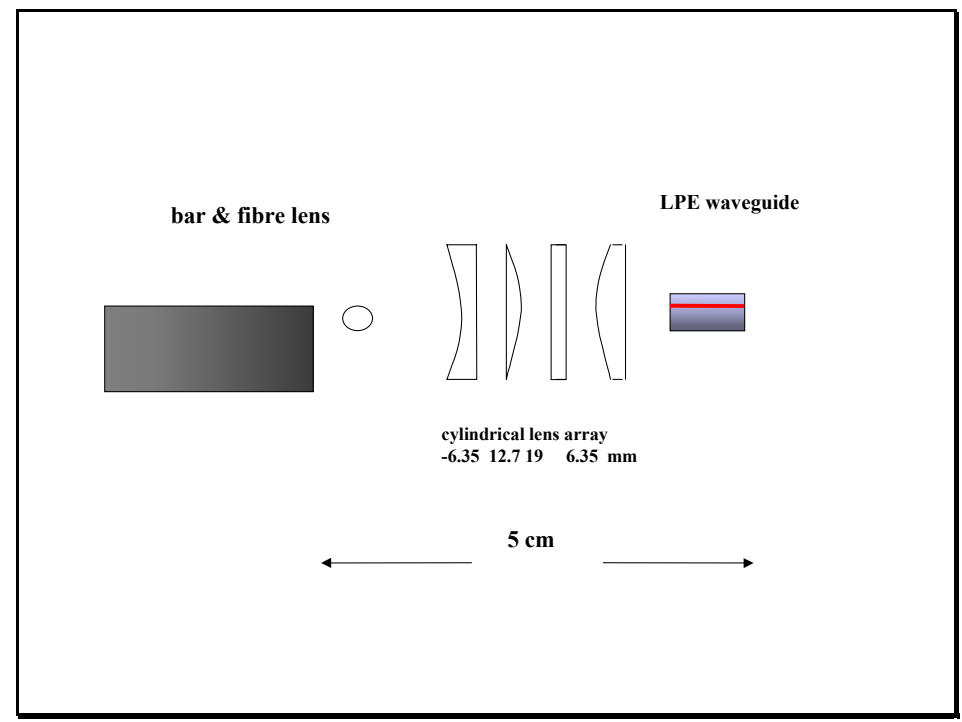

The output from the diode bar was collimated by a fibre lens into a sheet of radiation, $\sim 320 \mu \mathrm{m}$ deep and up to $18 \mathrm{~W}$ in power, with beam quality parameter values $\mathrm{M}_{\mathrm{x}}{ }^{2} \sim 2.3$ and $\mathrm{M}_{\mathrm{y}}{ }^{2} \sim 2300$. The waveguide was more highly multimode than the pump characteristics required; with a depth of $80 \mu \mathrm{m}$ it supported TE and TM laser modes up to $\mathrm{m}=8$, despite a low NA of 0.06 . The laser output power is shown as a function of diode operating current in Fig. 6. The greatest observed output power was $6.2 \mathrm{~W}$, corresponding to an overall optical-to-optical conversion efficiency of $31 \%$.

The output from this laser was in a multimode beam, with characteristics that were sensitive, for example, to the effectiveness of the contact between substrate and heatsink. $\mathrm{M}^{2}$-values were estimated for the beam based on intensity profile measurements. These ranged from $3-5$ in the vertical dimension and from $140-160$ in the lateral dimension. The best observed values of beam quality parameter therefore corresponded to a brightness enhancement of

$$
\left(\frac{6.2}{18}\right)\left(\frac{2.3 \times 2300}{3 \times 140}\right)\left(\frac{808}{1064}\right)^{2}=2.5
$$


relative to the fibre-lensed diode pump beam.

Figure 6: Planar waveguide laser output power at $1.064 \mu \mathrm{m}$ as a function of diode bar operating current.

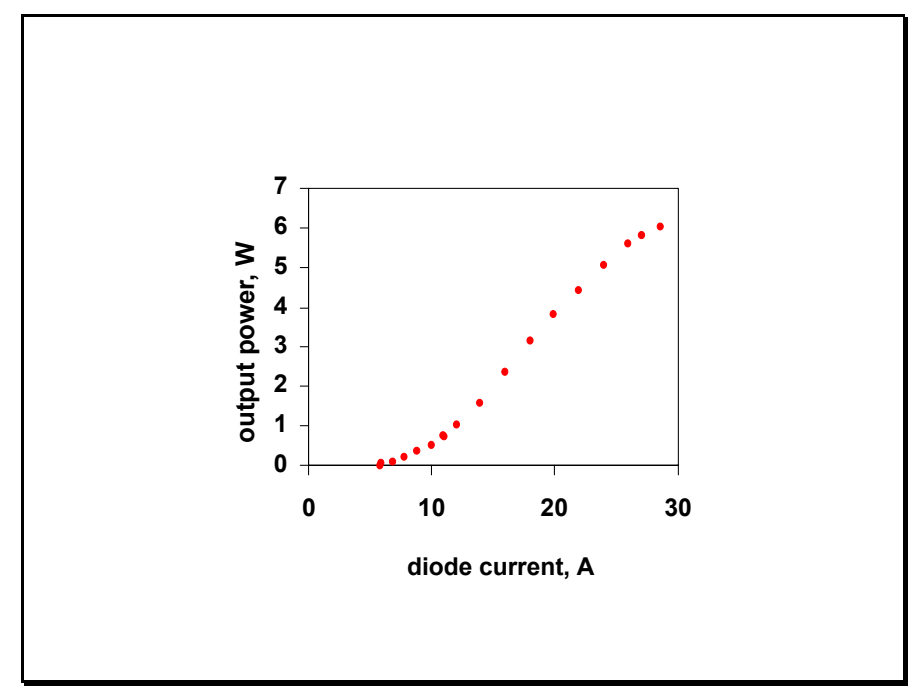

It was clear that the benefits of the guided wave geometry would only be fully exploited in these experiments if thin waveguides, of depth $<10 \mu \mathrm{m}$, were used. Such guides would need to have large NA to capture the highly diverging diode output efficiently. The first experiment of this type accordingly used a thermally bonded planar waveguide with a $\mathrm{Nd}$ :YAG core bounded by sapphire cladding regions. This combination of materials gives guides with NA $\sim 0.46$. Guides with depth as low as $4 \mu \mathrm{m}$ can be fabricated by contact bonding, with propagation losses measured to be less that $0.2 \mathrm{~dB} \mathrm{~cm}^{-1}$ in titanium sapphire laser-pumped lasing experiments. A particularly simple assembly of coupling optics was used to launch light from a $20 \mathrm{~W}$ diode bar into these guides. A single graded-index rod lens was used to launch the collimated sheet of radiation from the fibre-lensed bar into the guide, while a single $19-\mathrm{mm}$ focal length cylindrical lens was used to focus the light in the unguided lateral direction to a region about $4 \mathrm{~mm}$ wide. The distance from the output facet of the diode to the input face of the waveguide was $3 \mathrm{~cm}$. With this arrangement it was possible to couple $73 \%$ of the diode output into an $8-\mu \mathrm{m}$ deep guide, and $60 \%$ into a $4-\mu \mathrm{m}$ one (Shepherd et al., 1999). In a preliminary laser experiment an output power of $3.7 \mathrm{~W}$ was observed from the $8 \mu \mathrm{m}$ guide, although waveguide length and output coupling were not optimised in this device.

Most recently it has been shown that diode bars can be coupled efficiently into planar waveguides without any optical components at all - by proximity coupling (Bonner et al, 1999). Precision manipulators were used to position the emitting facet of a 10-W diode bar within $\sim 20$ $\mu \mathrm{m}$ of the end face of an 8- $\mu \mathrm{m}$ deep planar guide. A contact-bonded Nd:YAG-on-sapphire waveguide was used for this experiment, for the sake of the high NA of $\sim 0.45$. By measuring the total pump power transmitted through the 2-mm long structure, both guided and in radiation modes, and comparing it to that emitted by the diode, it was calculated that $45 \%$ of the incident power was absorbed in the waveguide. The efficiency of coupling into the waveguide was thereby estimated to be high, probably $\sim 90 \%$. The guide was observed to lase transversely, emitting about $0.5 \mathrm{~W}$ for $6 \mathrm{~W}$ of pump power, in a resonator that was far from being optimised. 
These results suggest that proximity coupling may form the basis for a family of highly compact and efficient sources, especially bearing in mind that position tolerances for a cladding-pumped device might be significantly relaxed.

\subsection{Mode control in planar laser resonators}

Since these sources are extremely compact, and potentially simple and efficient, it is important to investigate the extent to which their spatial mode quality can be improved. The guided and unguided directions present separate problems, and will be considered in turn.

\subsubsection{Mode-control in the guided direction}

Although it is often stated that for the fast-diverging plane of a diode bar $\mathrm{M}^{2}=1$, in practice the initial divergence is so great that after the fibre lens the $\mathrm{M}^{2}$-value is typically $2-3$. It may therefore not be possible to couple the pump light into a guide which is monomode at the laser wavelength. An interesting possibility for such devices is to control the quality of the spatial mode in the guided direction using the evanescent field. An experimental study has shown that gold overlays can be used to create excess propagation losses for higher order TE modes, with considerable enhancement of beam quality (Brown et al 1998). A 50-nm thick gold coating was applied to an LPE-grown guide consisting of an 8.3- $\mu$ m thick YAG layer doped with $\mathrm{Nd}$, Ga and Lu grown on an undoped YAG substrate. There was no superstrate, and the gold was applied directly to the upper surface of the core. The NA of the guide was 0.226 , so that the guide depth was equivalent to 1.8 scaling lengths, and the guide supported TE and TM modes up to $\mathrm{m}=3$. An experimental comparison was made between Au coated and uncoated regions of the guide, measuring the beam quality of the lasing output in the guided direction. It was possible to adjust the focusing of the titanium sapphire pump laser into the guide so that the higher order guided modes at the laser wavelength saw at least as much gain as the fundamental. Under these conditions whereas the output of the bare guide exhibited $\mathrm{M}^{2}$-values in the range $1.52-2.5$, the value from the gold-coated region was 1.17. The gold coating had the further effect of suppressing lasing on all TM modes, so that the output became linearly polarised, with the ratio of maximum to minimum values of power transmitted through a rotating linear polariser of 27 $\mathrm{dB}$, compared with $7 \mathrm{~dB}$ in the uncoated region. An analysis of propagation in this system showed that the gold layer introduced excess losses of only $0.04 \mathrm{~dB} / \mathrm{cm}$ for the fundamental TE mode, but $0.5 \mathrm{~dB} / \mathrm{cm}$ for the highest order TE mode, and $1 \mathrm{~dB} / \mathrm{cm}$ for the fundamental TM mode.

An alternative approach to the problem of mode control in the guided direction is to use the technique of cladding-pumping, to which planar thermally-bonded structures are particularly well adapted. Since the $\mathrm{M}^{2}$-value of an unsymmetrized bar pump beam is quite low in the vertical direction, the inner cladding depth need only be $2-3$ times that of the core, so that the overall device length can be kept down to a few $\mathrm{mm}$. Thermal bonding is particularly wellsuited to assembling structures in which the NA of the core/inner cladding interface is small, whereas that of the inner cladding/outer cladding interface is large. The doped YAG/undoped YAG/sapphire material combination, with its low loss, mechanical strength and high thermal conductivity is at the time of writing under study as a particularly interesting system for this purpose.

\subsubsection{Mode-control in the unguided plane}


The planar laser devices which have been constructed to date typically have $\mathrm{M}^{2}$-values of $100 \mathrm{or}$ more in the unguided plane; this is clearly the characteristic which is in most urgent need of improvement. The plane/plane Fabry-Perot resonator used hitherto does little for the lateral spatial coherence; however it is desirable to keep the compact and rugged monolithic character of these devices rather than incorporate an external resonator.

It is evident that a significant reduction in $\mathrm{M}^{2}$-value can be achieved by restricting the aperture for laser emission. Planar waveguide lasers with lateral $\mathrm{M}^{2}$ of $\sim 30$ have been made by orienting the cavity at right angles to the direction of the incident pump beam. In this side-pumped geometry the width of the gain medium can be substantially reduced, though at the expense of a very much smaller overlap between the pump and laser modes. In an alternative approach the structure shown in Fig. 6 was constructed, with a sliding output mirror controlling the variable width of the emission aperture. With this type of cavity output power was cut down from $3.7 \mathrm{~W}$ to $2.4 \mathrm{~W}$, but with considerable improvement in $\mathrm{M}^{2}$, from 85 to 22 in the unguided direction. A future solution to the problem of lateral beam quality in planar waveguide lasers is therefore likely to be the monolithic planar unstable resonator. Such a device might incorporate cladding pumping to give an output of several $\mathrm{W}$, in a beam with $\mathrm{M}^{2}$-values of 2-3 in the lateral direction and $<1.2$ in the guided direction.

\subsection{Face-pumped lasers}

Although side-pumping, with the pump laser light propagating across the laser resonator, suffers in efficiency compared to longitudinal pumping because the overlap of pump and signal is unavoidably weak, it has the attractive property of scalability. The power of a side-pumped device can in principal be increased up to the limit set by optical damage simply by multiplying up the length of the gain medium and the pump units coupled to it; the thermal load along the extended gain medium can everywhere be the same. Pumping through the face of a planar structure is equally scalable, and has the further highly attractive feature that the pump light is not launched into a waveguide, and positioning with micron accuracy is not required.

A fundamental difficulty attending face-pumped lasers, whether waveguide or slab, is the weak absorption of pump light by typical dielectric gain media. Any attempt to exploit face pumping in these systems therefore involves multiple passes of the pump through the gain medium. We shall examine two such schemes. In the first, due to Brauch et al. (1994), a thin Yb:YAG disc plays the role of an amplifying mirror in an otherwise conventional laser cavity. Diode bar pump light is delivered by a fibre bundle, and an array of mirrors re-image the reflected pump back on to the active area of the device. Heat can be removed with great efficiency from the thin slab, and thermal gradients are to first order directed along the axis of the laser cavity, greatly reducing their adverse effect on the quality of the output beam. Many tens of watts can be extracted from these devices in beams of excellent quality; the most difficult aspect of the design is the alignment of the multiple pump mirrors.

In the second scheme the laser light propagates in the plane of the pumped face, and a slotted mirror achieves the necessary multipass pumping (Faulstich, Baker and Hall, 1996). The pump source is a stack of diode bar lasers, their emitting regions aligned immediately behind slots in a highly reflecting mirror. The gain medium, a slab of Nd-doped glass, is proximity-coupled to the 
pump, the rapid divergence of the uncollimated diode beams ensuring a uniform distribution of pump energy. A second reflecting surface below the slab sets up the multiple pump relflections, and spaces either side allow the passage of coolant. In this way a transfer efficiency of the pump light of $84 \%$ has been achieved into a slab only $0.45 \mathrm{~mm}$ thick with $32 \%$ single-pass absorption of the pump. Pulses of $65 \mathrm{~mJ}$ have been extracted from this system. The slotted mirror technique has also been used to pump a multimode thermally-bonded Nd:YAG on YAG waveguide, which emitted $9.3 \mathrm{~W}$ average power at $8 \%$ duty cycle.

It is finally worth noting that the limitation of weak pump absorption which motivates these elaborate multipass schemes is absent in semiconductor gain media, in which absorption coefficients are measured in $\mu \mathrm{m}^{-1}$ rather than $\mathrm{cm}^{-1}$. It is interesting that the active mirror concept embodied in the $\mathrm{Yb}$ :YAG disc laser described above has also been employed in an opticallypumped semiconductor quantum well laser, from which $\sim 0.5 \mathrm{~W}$ of output power was extracted in a diffraction-limited beam (Kuznetsov et al, 1997). The semiconductor wafer in this device incorporated 14 InGaAs quantum wells pumped by optical absorption in the barriers; a Bragg grating structure provided one mirror of the resonator, which was completed by an external spherical mirror. The laser design shares many features with its $\mathrm{Yb}$ :YAG counterpart, however the gain medium absorbs $\sim 85 \%$ of the incident pump light in a single pass.

It is clear that planar geometries, whether or not guided-wave, are particularly well adapted to high power pumping by low-brightness diode bar sources. The most attractive designs will be simple and compact and will not involve excessively tight alignment tolerances. Proximity coupling, in various guises, scores highly by these criteria, and this may be a prominent theme of future work. Moreover the optical pumping of semiconductors - long derided as the last resort of electrically defective structures - may turn out to be significant, bringing a new lease of life to designs developed for high power dielectric lasers through the great versatility of quantum well gain media.

\section{4. $\quad$ References}

Allain J.Y., Monerie M. and Poignant H., 1991, Electron. Lett. 27, 1156.

Anderson A.A., Eason R.W., Hickey L.M.B., Jelinek M., Grivas C. and Gill D.S., 1997, Opt. Lett. 22, 1556.

Barber P.R., Paschotta R., Tropper A.C., and Hanna D.C., 1995, Opt. Lett. 20, 2195.

Birks T.A., Farwell S.G., Russell P.St.J. and Pannell C.N., 1994, Opt. Lett. 19, 1964.

Bonner C.L., Anderson A.A., Eason R.W., Shepherd D.P., Gill D.S., Grivas C. and Vainos N., 1997, Opt. Lett. 22, 988.

Bonner C.L., Brown C.T.A., Shepherd D.P., Clarkson W.A., Tropper A.C., Hanna D.C. and Ferrand B., 1998. Opt. Lett. 23, 942.

Bonner C.L., Bhutta T., Shepherd D.P., Tropper A.C., Hanna D.C. and Meissner H.E., 1999, Proceedings CLEO-US. 
Booth I.J., Archambault J.-L. and Ventrudo B.F., 1996, Opt. Lett. 21, 348.

Brauch U., Giesen A., Karszewinski M., Stewen C. and Voss A., 1995, Opt. Lett. 20, 713.

Brown C.T.A., Bonner C.L., Warburton T.J., Shepherd D.P., Tropper A.C., Hanna D.C. and Meissner H.E., 1997, Appl. Phys. Lett. 71, 1139.

Brown C.T.A., Harris R.D., Shepherd D.P., Tropper A.C., Wilkinson J.S. and Ferrand B., 1998, Phot. Tech. Lett. 10, 1392.

Culverhouse D.O., Richardson D.J., Birks T.A. and Russell P.St.J., 1995, Opt. Lett. $20,2381$.

Daran E., Shepherd D.P. and Schweizer T., 1998, Proceedings of CLEO-EUROPE, paper CThF7.

Desurvire E., 1994, Erbium-doped fiber amplifiers: principles and applications, John Wiley $\&$ sons.

Digonnet M.F.J. and Gaeta C.J., 1985, Applied Optics 24,333.

Faulstich A., Baker H.J. and Hall D.R., 1996, Opt. Lett. 21, 594.

Funk S. and Eden J.G., 1995, IEEE J. Sel. Topics Quantum Electronics 1, 784.

Grubb S.G., Bennet K.W., Cannon R.S. and Humer W.F., 1992, Electron. Lett. 28, 1243.

Guy S., Bonner C.L., Shepherd D.P., Hanna D.C., Tropper A.C. and Ferrand B., 1998, IEEE J. Quantum Electronics, 34, 900.

Hickey L.M.B, Quigley G.R., Wilkinson J.S., Moya E.G., Moya F. and Grattepain C., 1998, Proceedings CLEO-EUROPE.

Hill K.O., Malo B., Bilodeau F., Johnson D.C. and Albert J., 1993, Appl. Phys. Lett. 62,1035 .

Kringlebotn J.T., Archambault J.-L., Reekie L. and Payne D.N., 1994, Opt. Lett. 19, 2101.

Kuznetsov M., Hakimi F., Sprague R. and Mooradian A., 1997, IEEE Phot. Tech. Lett. 9, 1063.

Laperle P., Chandonnet A. and Vallée R., 1995, Opt. Lett. 20, 2484. 
Miniscalco W.J. and Quimby R.S., 1991, Opt. Lett., 16, 258.

Muendel M., Engstrom B., Kea D., Laliberte B., Minns R., Robinson R., Rockney B., Zhang Y., Collins R., Gavrilovic P. and Rowley A., 1997, Postdeadline paper CPD30-1, CLEO-US 1997.

Nishihara H., Haruna M., and Suhara T., 1985, Optical Integrated Circuits, McGraw-Hill.

Paschotta R., Moore N., Clarkson W.A., Tropper A.C., Hanna D.C. and Mazé G., 1997, IEEE J. Sel. Topics Quantum Electronics 3, 1100.

Pelenc D., Chambaz B., Chartier I., Ferrand B., Wyon C., Shepherd D.P., Hanna D.C., Large A.C. and Tropper A.C., 1995, Opt. Comm. 115, 491.

Peto A., Townsend P.D., Hole D.E. and Harmer S., 1997, J. Mod. Optics 44, 1217.

Richardson D., Minelly J. and Hanna D.C., 1997, Laser Focus World, Sep. 87.

Sanders S., Waarts R.G., Mehuys D.G. and Welch D.F., 1995, Appl. Phys. Lett. 67, 1815.

Shepherd D.P., Brown C.T.A., Warburton T.J., Hanna D.C. and Tropper A.C., 1997, Appl. Phys. Lett. 71, 876.

Shepherd D.P., Bonner C.L., Brown C.T.A., Clarkson W.A., Tropper A.C., Hanna D.C., Meissner H.E., 1999, Opt. Comm. 160, 47

Smart R.G., Hanna D.C., Tropper A.C., Davey S.T., Carter S.F. and Szebesta D., 1991, Electron. Lett. 27, 1309.

Snitzer E., 1961, Phys. Rev. Lett. 7, 444.

Snyder A.W. and Love J.D., Optical Waveguide Theory, 1983, Chapman and Hall.

Suche H., Wessel R., Westenhöfer S., Sohler W., Bosso S., Carmannini C. and Corsini R., 1995, Opt. Lett. 20, 596.

Townsend P.D., Chandler P.J. and Zhang L., 1990, Optical Effects of Ion Implantation, Cambridge University Press.

Wang J., Hector J.R., Brady D., Hewak D., Brocklesby B., Kluth M., Moore R. and Payne D.N., 1997, Appl. Phys. Lett. 71, 1753.

Xie P. and Gosnell T.R., 1995, Opt. lett. 20, 1014.

Yeung Y.Y. and Newman D.J., 1985, J. Chem. Phys., 82, 3747. 
